\title{
Erratum to: "Selective separation of some ecotoxic transition metal ions from aqueous solutions using immobilized macrocyclic material containing solid phase extraction system"
}

Erratum

Ismail M. M. Rahman ${ }^{1,2^{*}}$, Yoshiaki Furusho ${ }^{3 * *}$, Zinnat A. Begum ${ }^{1}$,

Akhmad Sabarudin ${ }^{4,5}$, Shoji Motomizu ${ }^{6}$, Teruya Maki ${ }^{1}$, Hiroshi Hasegawa ${ }^{1^{* * * * * *}}$

'Graduate School of Natural Science and Technology,

Kanazawa University, Kakuma, Kanazawa 920-1192, Japan

'Department of Chemistry, Faculty of Science,

University of Chittagong, Chittagong 4331, Bangladesh

${ }^{3}$ GL Sciences Inc., Nishishinjuku 6-22-1, Shinjuku, Tokyo 163-1130, Japan

${ }^{4}$ Division of Nanomaterial Science, EcoTopia Science Institute,

Nagoya University, Furo-cho, Chikusa-ku, Nagoya 464-8603, Japan

${ }^{5}$ Department of Chemistry, Faculty of Science,

Brawijaya University, JI. Veteran Malang 65145, Indonesia

${ }^{6}$ Graduate School of Natural Science and Technology,

Okayama University, Tsushimanaka 3-1-1, Okayama 700-8530, Japan

Received 12 September 2011; Accepted 16 September 2011

Abstract: The original version of the article was published in Cent. Eur. J. Chem. 9(6) (2011), pp 1019-1026. Unfortunately, the original version of this article contains mistakes in the body of Fig. 1. Here we display the corrected version of the Fig. 1.

Keywords: Solid phase extraction • Molecular recognition technology • Non-destructive • Selective separation • Aqueous system (C) Versita Sp. z 0.0 .

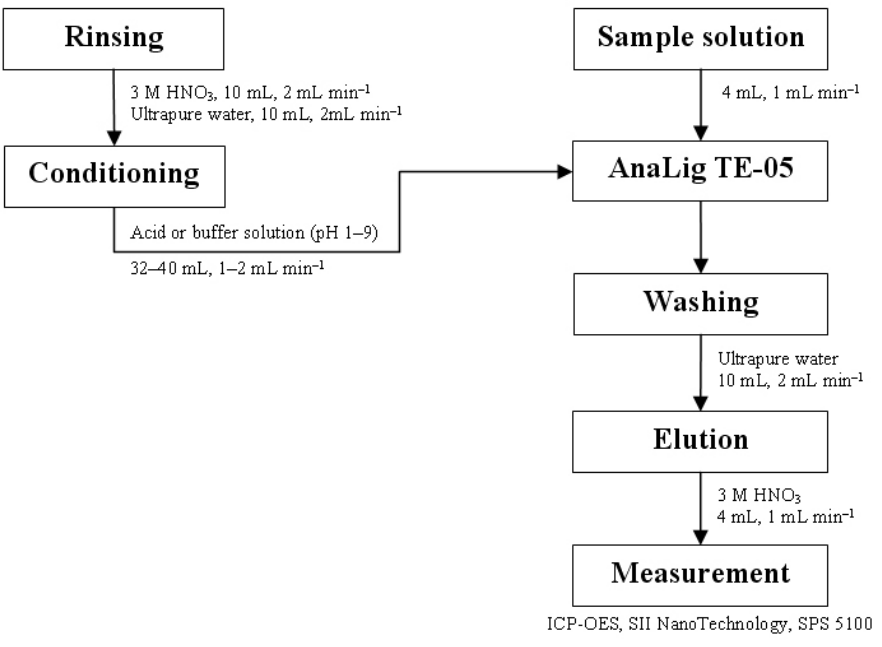

Figure 1. Schematic diagram of the experimental setup. 